\title{
Establishment of an animal model of intrauterine adhesions after surgical abortion and curettage in pregnant rats
}

\author{
Qing Feng", Bingsi Gao", Xingping Zhao, Huan Huang, Shuijing Yi, Lingxiao Zou, Xinyi Liu, Min Xue, \\ Dabao Xu \\ Department of Obstetrics and Gynecology, Third Xiangya Hospital of Central South University, Changsha 410013, China \\ Contributions: (I) Conception and design: M Xue, D Xu; (II) Administrative support: D Xu; (III) Provision of study materials or patients: Q Feng, B \\ Gao, X Zhao, H Huang, L Zou, X Liu; (IV) Collection and assembly of data: Q Feng, B Gao, S Yi; (V) Data analysis and interpretation: Q Feng, X \\ Zhao; (VI) Manuscript writing: All authors; (VII) Final approval of manuscript: All authors. \\ \#These authors contributed equally to this work. \\ Correspondence to: Dabao Xu, MD; Min Xue, MD. Department of Obstetrics and Gynecology, Third Xiangya Hospital of Central South University, \\ Changsha 410013, China. Email: dabaoxu@yahoo.com; Xuemin5908@sina.com.
}

Background: Intrauterine adhesion (IUA), also referred to as Asherman syndrome, is a complication with partial or complete adhesion of the uterine cavity due to fibrotic proliferation after endometrial and uterine wall injury of the newly pregnant uterus. Currently, all IUA research models are based on non-pregnant animals which are ill-suited in mimicking its pathogenesis. This study aimed to establish an animal IUA model caused by surgical abortion and curettage in pregnant rats that is more in line with clinical etiology, and to provide a more ideal animal model for further exploration of the pathogenesis and treatment for IUA. Methods: Fifteen, 13-to-15-day pregnant Sprague-Dawley (SD) rats aged 10 weeks were selected. After incising and removing the embryos, we randomly selected one side of the uterine cavity as the study group $(n=15)$ and scraped it with a curette; the contralateral side of the uterine cavity that underwent no special procedures was used for inclusion in the control group $(n=15)$. Five rats were euthanized and dissected on the $3 \mathrm{rd}$, 7th, and 14th day after surgery respectively. The changes in tissue morphology, the number of endometrial glands, the ratio of endometrial fibrotic area, and the expression level of the transforming growth factor beta (TGF- $\beta$ ) in the endometrium were compared between the 2 groups.

Results: The endometrial granular cell number in the study group on the 7 th and 14th day postoperatively was $3.87 \pm 0.72 \& 2.59 \pm 0.90$ in each visual field $(\times 100)$ respectively, which was significantly lower than that of the control group $(6.48 \pm 0.96 \& 7.53 \pm 1.10 ; \mathrm{P}<0.05)$. The ratio of the endometrial stromal fibrotic area in the study group was significantly higher than that of the control group post-operatively on the 3 rd day $(0.0974 \pm 0.0430$ vs. $0.0584 \pm 0.05110)$, 7 th day $(0.1551 \pm 0.0348$ vs. $0.0328 \pm 0.0152)$, and 14 th day $(0.1518 \pm 0.0477$ vs. $0.0311 \pm 0.0128)(\mathrm{P}<0.05)$. The endometrial TGF- $\beta$ expression in the study group was positive on the 14th day postoperatively while weakly expressed in the control group, and the integrated optical density (IOD) value $(5,608.8 \pm 2,887.3)$ was higher than that of the control group $(388.3 \pm 98.6)(\mathrm{P}<0.05)$. The endometrial TGF- $\beta$ expression in the study group and control group was relatively weak on the $3 \mathrm{rd}$ and 7 th day after surgery, and there was no significant difference when compared with that of the control group $(\mathrm{P}>0.05)$.

Conclusions: The establishment of an animal model of IUA after surgical abortion and curettage in pregnant rats was successful and is thus feasible for further studies; the model may be a more effective mimic for the generation of human IUA.

Keywords: Intrauterine adhesions (IUAs); animal models; pregnant rats; endometrial fibrosis; pathogenesis

Submitted Dec 20, 2019. Accepted for publication Jan 14, 2020.

doi: 10.21037/atm.2020.01.134

View this article at: http://dx.doi.org/10.21037/atm.2020.01.134 


\section{Introduction}

Intrauterine adhesion (IUA), also referred to as Asherman syndrome, is a complication of partial or full adhesion in the uterine cavity due to fibrous tissue proliferation after uterine wall and endometrial injury during pregnancy $(1,2)$. IUA can not only cause menstrual disorders including oligomenorrhea, amenorrhea and periodic lower abdominal pain, but also fertility disorders such as infertility, abortion, and placental abnormalities (3). Although IUA was first described by Heinrich Fritsch as early as 1894, the pathogenesis, symptoms evolution, prevention, and treatment strategies of IUA have not been fully explained.

Since the 1970s, researchers have used various methods to establish an animal IUA model, including physical injury, chemical injury, mechanical injury, and a dual pathological injury model combining two methods $(4,5)$. The endometrium tends to regenerate and repair shortly after single mechanical injury, and, with this strong endometrial regeneration of experimental animals, it is difficult to establish an effective IUA animal model. Consequently, researchers have explored combining 2 or more injury methods for the establishment of IUA animal models (6). For example, a dual pathological injury model was achieved by removing the ovaries or inducing intrauterine infections using bacterial lipopolysaccharide based on dilatation and curettage (D\&C) (7). There are also rat chemical pathological injury models and rat physical pathological injury models $(4,5)$. However, all these models are based on non-pregnant animals, and there is a difference in the endometrial damage caused by either physical or chemical methods resulting in adhesion and the actual clinical causes of IUA patients along with subsuquent pathological changes of IUA in vivo. Thus, the simulatation and related research and treatment methods for the occurrence mechanism of human IUA cannot claim complete accuracy or fidelity (8). Therefore, it is necessary to explore the establishment of an animal model which is more in line with the mechanism of human IUA.

In this study, we created and identified an animal IUA model using surgical abortion and curettage in pregnant rats which is closer to the clinical etiology. Using this method, we hope to provide a more ideal animal model for further exploration of the pathogenesis and treatment for IUA.

\section{Methods}

\section{Animals}

Fifteen 13-to15-day pregnant Sprague-Dawley (SD) rats aged 10 weeks and weighing between $400-450 \mathrm{~g}$ were selected. They were purchased from Hunan SJA Laboratory Animal Co., Ltd . All animals were housed individually with free access to a standard diet and clean water in a temperature- $\left[(23 \pm 2)^{\circ} \mathrm{C}\right]$ and light- $(12 \mathrm{~h} \mathrm{light}$ and $12 \mathrm{~h}$ dark $)$ controlled environment before surgery. All experimental procedures were approved by the Animal Experimentation Ethics Committee of Third Xiangya Hospital of Central South University, Hunan, China.

\section{Establishment of animal models}

Pregnant rats were anesthetized with an intraperitoneal injection of chloral hydrate $(100 \mathrm{~g} / \mathrm{L})$ at a dose of $3 \mathrm{~mL} / \mathrm{kg}$. All the operations were performed by the same investigator. The details of the procedures are as follows: after disinfection with complexed iodine, a 4-cm longitudinal midline incision of the lower abdomen was made to access the abdominal cavity, and the uterus was exposed on both sides; then, a 1-cm longitudinal uterine incision was made close to the proximal end of the uterus, and the bilateral embryos were removed intactly; 1 side of the uterine cavity was randomly selected (study group), and the rat endometrium was scraped with a curette in 4 different directions (front, back, left, and right); the contralateral uterine cavity was included in the control group, and no special procedures were performed after confirming that the embryo was completely removed; the uterine incision and abdominal wall incision were then sutured (Figure 1A,B,C).

\section{Identification methods of IUA model in pregnant rats}

Five rats were individually euthanized and dissected on the 3rd, 7th, and 14th days after surgery respectively. The changes in tissue morphology, the number of endometrial glands, the degree of endometrial fibrosis, and the expression level of the transforming growth factor beta (TGF- $\beta$ ) in the endometrium were compared between the study group and control group.

\section{Histologic examination with hematoxylin and eosin (H\&E) staining}

The dissected uterus was fixed in $4 \%$ formaldehyde and sectioned into paraffin $24 \mathrm{~h}$ later. The sections were serially sliced at $4 \mu \mathrm{m}$. Three sections were randomly selected from each rat uterus and routinely stained with H\&E. Singleblinded slide reading was performed by the same experienced pathologist. Each H\&E slice was randomly selected into 

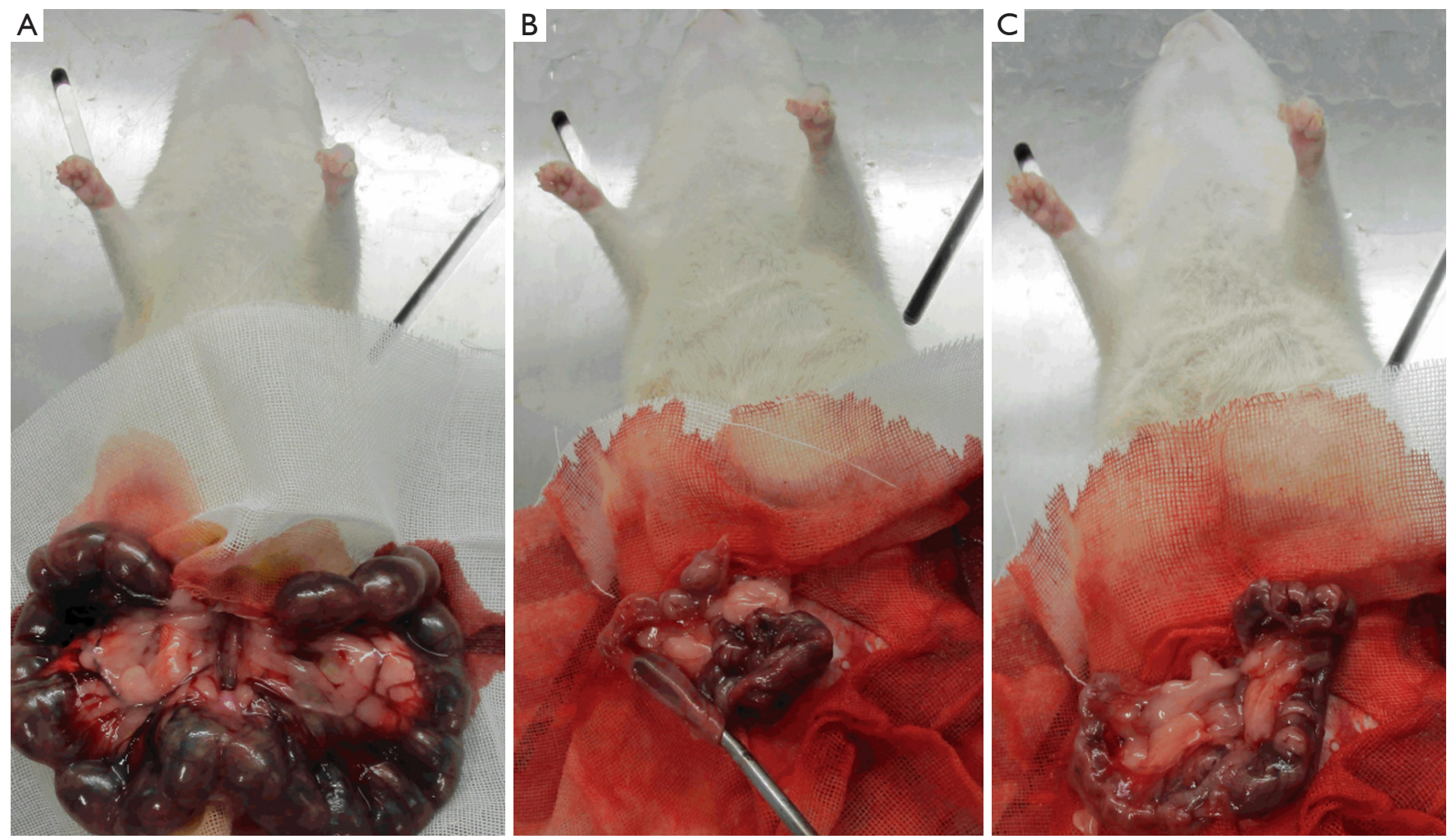

Figure 1 Main surgical procedures. (A) Uterus exposure; (B) endometrial abrasion curettage with a curette; (C) uterine suture and abdominal wall incision.

4 visual fields under a $50 \times$ microscope. The number of endometrial glands in each visual field was counted, and the average value was recorded for future statistics.

\section{Detection of endometrial fibrosis}

All the rat uteruses were embedded in paraffin in the same way as described above, and $4 \mu \mathrm{m}$ serial sections were created. Three sections of each rat uterus were randomly selected for Masson staining, 4 fields of each Masson slice were randomly selected under high magnification $(\times 100)$, and the image analysis system Image-Pro Plus 6.0 was used to calculate the area of endometrial stromal fibrosis, which was the total area of the endometrium and glands in the selected microscopic field. The fibrosis ratio was calculated as the area of the endometrial stromal fibrosis in each field divided by the total area of the endometrial interstitium and glands, with the average value being recorded for further statistical analysis.

\section{Immunohistochemistry (IHC)}

The specimen sections were dewaxed and hydrated. After high-temperature and high-pressure antigen repair, they were incubated with $3 \% \mathrm{H}_{2} \mathrm{O}_{2}$ at room temperature for 10 min to block endogenous peroxidase. TGF- $\beta$ (AY0411, ABZOOM) primary antibody was added at a dilution of 1:200 and incubated at $4{ }^{\circ} \mathrm{C}$ overnight. Phosphate-buffered saline (PBS) was used as the negative control. The specimen was removed and rewarmed at room temperature for $30 \mathrm{~min}$ after overnight incubation at $4{ }^{\circ} \mathrm{C}$ in a wet box. Horseradish peroxidase (HRP)-labeled polymer (ready-to-use) (P009IH, Auragene) was added and incubated at room temperature for $30 \mathrm{~min}$ after washing with PBS. After being incubated with 3,3'-Diaminobenzidine (DAB) at room temperature for 30 s, the specimen sections were counterstained by hematoxylin. Slides were dehydrated with alcohol, cleared with xylene, and sealed with neutral gum. Three highpower fields were randomly selected under each high-power lens $(\times 200)$ for each section, and there was no blank area in each field. The endometrial IHC staining section images were captured and measured using the image analysis software Image-Pro Plus 6.0. For each picture's integrated optical density (IOD), each slice was randomly observed 
in 4 fields of view, and the IOD value of the endometrial protein was calculated. The average value was collected as the IOD value of the uterine specimen.

\section{Statistical analysis}

The data were processed with SPSS 20.0 software, and the $t$-test was used to compare the number of glands, the ratio of endometrial fibrosis area, and the IOD value. The difference was considered statistically significant with $\mathrm{P}<0.05$ and highly statistically significant with $\mathrm{P}<0.01$.

\section{Results}

\section{Establishment of the model}

Fifteen pregnant rats were processed for the initial IUA model establishment, 14 survived, and 1 died because of excessive bleeding on the first postoperative day; the survival rate was $93.3 \%$. We followed the experimental procedure and re-processed another pregnant rat for the supplement. The dissection of the last rat was strictly and separately processed, according to the designed check point.

\section{Identification of the model}

\section{Observation of endometrial H\&E staining and} comparison of the endometrial glandular cell number

On the 3rd day after curettage of the pregnant rats, there were thin endometria, sparse distribution of glands, proliferated interstitial cells, edema, proliferated and congested small blood vessels, and infiltrated inflammatory cells observed in the control group. When compared with the control group, the thickness of the endometrium of the study group was reduced, and the endometrial glandular number was slightly reduced $(\mathrm{P}>0.05)$. Submucosal fibrosis, inflammatory cell infiltration, and some capillaries expansion and congestion could also be found in the study group.

On the 7 th and 14th day after surgery, the endometrium gradually became thickened and the number of glands increased in the control group $(6.48 \pm 0.96,7.53 \pm 1.10$, respectively), with occasional fibrous hyperplasia. While in the study group, the number of endometrial glands continued to decrease on post-operative day $7(3.87 \pm 0.72$, significantly different from the control group, $\mathrm{P}<0.05$ ), while the number of fibrocytes and fibroblasts increased, with an enrichement of inflammatory cell infiltration. On the 14th day after surgery, some muscle fibrous adhesions could be seen in the uterine cavity, and the distribution of subepithelial glands was extremely sparse $(2.59 \pm 0.90$, significantly statistically different compared to the control group, $(\mathrm{P}<0.05)$. The interstitial fibroblasts were significantly proliferated $(\mathrm{P}<0.05)$, with little inflammatory cell infiltration (Figure $2 A, B$ ).

\section{Comparison of the degree of endometrial fibrosis}

To assess the degree of fibrosis of the damaged endometrium in the IUA model, we performed Masson staining of collagen fibers to detect endometrial fibrosis. Masson staining microscopy showed that the endometria of the control group were intact; the mucosa, submucosa, blood vessels, and smooth muscles were stained dark red; the endometrial stroma was uniformly edema-free; and the stromal collagen fibers were blue, fusiform, and aligned.

However, in the study group, the number of collagen fibers increased in some areas on the 3rd day after surgery. On the 7 th day post-operatively, some fibrous adhesions were seen in some uterine cavities, and the number of collagen fibers under the epithelium were increased. On the 14th day after surgery, the distribution of subepithelial glands were sparse, stromal fibrous tissue increased, collagen was aggregated, fibrosis was more obvious, and even muscular adhesions could be found in some uterine cavities (Figure $3 A$ ). The ratio of the endometrial stromal fibrotic area in the study group was significantly higher than that of the control group on post-operative day 3 $(0.0974 \pm 0.0430$ vs. $0.0584 \pm 0.05110)$, day $7(0.1551 \pm 0.0348$ vs. $0.0328 \pm 0.0152)$, and day $14(0.1518 \pm 0.0477$ vs. $0.0311 \pm 0.0128)(\mathrm{P}<0.05)$ (Figure $3 B)$.

\section{IHC results}

TGF- $\beta$ is mainly expressed in the nucleus and cytoplasm of stromal cells and epithelial cells, but it plays a vital role in endometrial fibrosis in uterine adhesions. Therefore, TGF- $\beta$ IHC was used to assess the severity of IUA in animal models. The endometrial TGF- $\beta$ expression in the study group was positive on the 14th day postoperatively while weakly expressed in the control group (Figure $4 A$ ), and the IOD value $(5,608.8 \pm 2,887.3)$ was higher than that of the control group $(388.3 \pm 98.6)$, with the difference being statistically significant $(\mathrm{P}<0.05)$. The endometrial TGF- $\beta$ expression in the study group was relatively weak on the $3 \mathrm{rd}$ and 7 th day after surgery, and there was no significant difference when compared with the control group $(\mathrm{P}>0.05)$ (Figure 4B). 

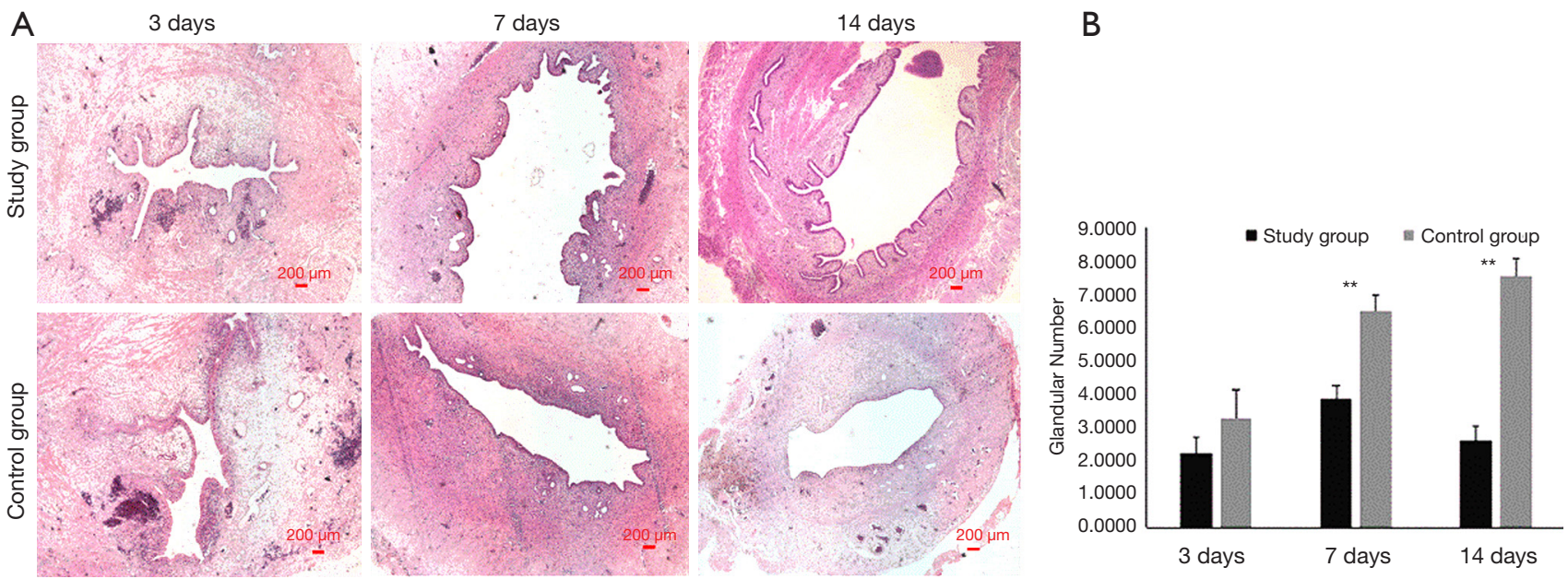

Figure 2 H\&E-stained sections of SD rat uterine tissue after curettage. (A) Post-operative days 3, 7, and 14 of the rat endometria from the 2 groups 1 Bar $=200 \mu \mathrm{m}$. (The study group: Day 3: the number of endometrial glands was slightly reduced, and submucosal fibrous hyperplasia could be seen. Day 7: there was a reduced number of endometrial glands and an increased number of fibroblasts and fibroblasts. Day 14: muscle fibrous adhesions could be observed. The control group: Day 3: the endometrium was thin with few fibroblasts. Days 7 and 14: the endometrium gradually thickened, and the number of glands increased). (B) The comparison of the endometrium glandular number between the 2 groups. [There was no significant difference between the 2 groups on post-operative day 3 ( $\mathrm{P}>0.05)$; however, the study group had a significantly lower glandular number than the control group on day 7 and day 14 ; **, $\mathrm{P}<0.001]$. H\&E; hematoxylin and eosin; SD, SpragueDawley;
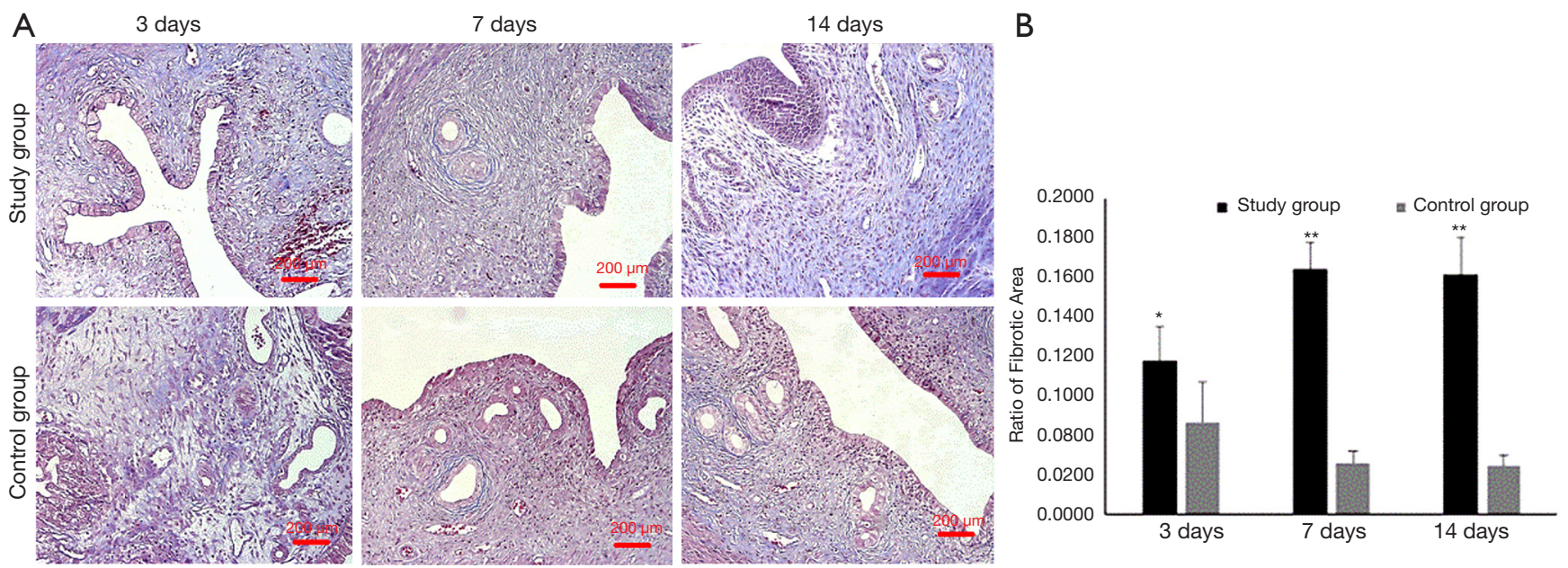

Figure 3 Masson sections of rabbit uterine tissue after curettage. (A) Post-operative days 3, 7, and 14 rat endometria from the 2 groups; Bar $=200 \mu \mathrm{m}$. (The study group: Day 3: partial collagen fibers increased. Day 7: some fibrous adhesions could be seen in uterine cavities, and collagen fibers under the epithelium increased. Day 14: obvious fibrosis and muscle adhesions could be seen in some uterine cavities. The control group: Endometrial structures were intact, and the stromal collagen fibers were arranged neatly). (B) The comparison of the stromal fibrosis area of the endometrium between the study group and control group. (Days 3, 7, and 14: the stromal fibrosis area of the endometrium for the study group was significantly greater than that of the control group; $\left.{ }^{*} \mathrm{P}<0.05 ;{ }^{* *}, \mathrm{P}<0.001, \mathrm{n}=5\right)$. 

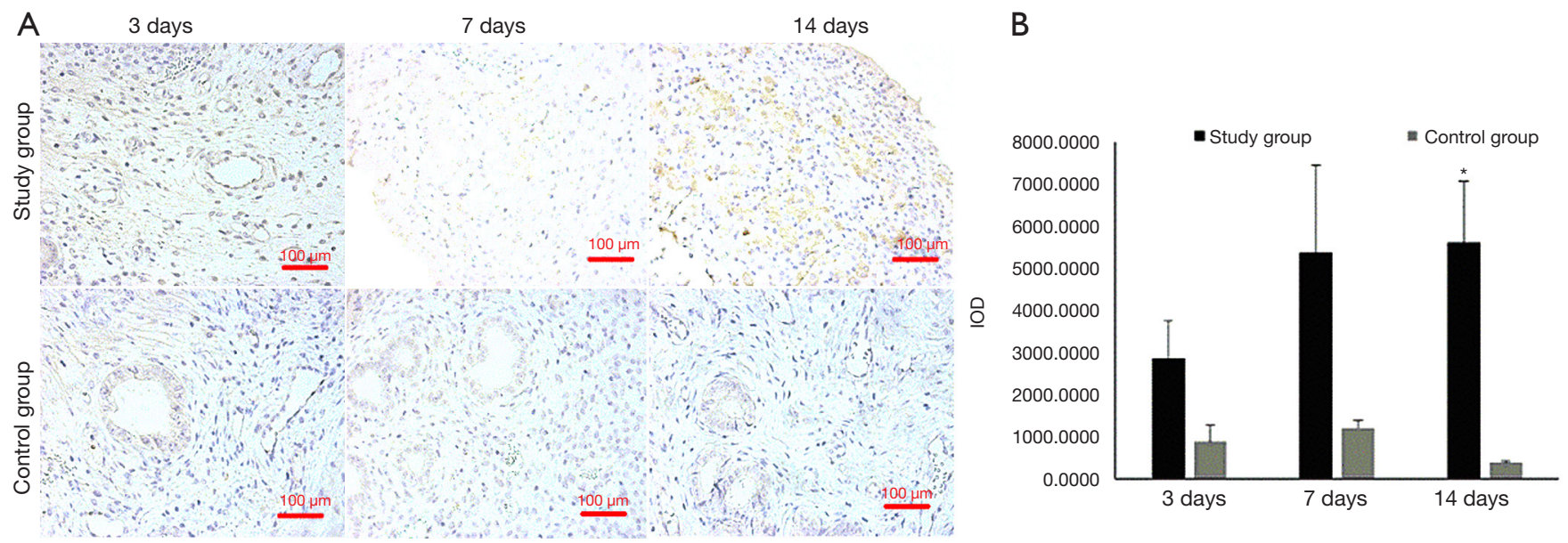

Figure 4 TGF- $\beta$ expression level of SD rat endometrium. (A) Post-operative day 3, 7, and 14 rat endometria from the 2 groups; Bar $=200 \mu \mathrm{m}$. (The study group: Day 3 and 7: there was weak endometrial TGF- $\beta$ expression. Day 14: there was positive endometrial TGF- $\beta$ expression. The control group: there was weak endometrial TGF- $\beta$ expression on days 3 , 7 , and 14 ). (B) The TGF- $\beta$ IOD value comparison between the study group and control group. (There was no significant difference between the 2 groups on day 3 and day 7; P>0.05), while the study group had a statistically significant higher TGF- $\beta$ expression level than that of the control group on day $14 ;$ *, $\mathrm{P}<0.05$; $\mathrm{n}=5$ ). TGF- $\beta$, transforming growth factor beta; SD, Sprague-Dawley; IOD, integrated optical density.

\section{Discussion}

\section{Main findings}

In this study, an IUA animal model was established using the method of curettage with pregnant rats according to the main risk factors of IUA, which is coincident with the clinical manifestation. We found that on the 3 rd day after the curettage of pregnant rats, the endometrial fibers were proliferated. On the 7 th day after the procedure, the endometria became thinner, the endometrial glands decreased from $6.48 \pm 0.96$ to $3.87 \pm 0.72$ and the degree of fibrosis increased from $0.1551 \pm 0.0348$ to $0.0328 \pm 0.0152$ when compared to the control group. The endometrial glands continued to decrease to $2.59 \pm 0.90$ on day 14 , with significant fibrosis and increased expression of TGF- $\beta$. This indicates that the method of curettage using pregnant rats can successfully establish an animal model of IUA.

\section{Previous research}

To study the occurrence, development, and treatment of IUA, many researchers have tried various methods to establish animal IUA models since the 1970s. In 1975, Polishuk et al. (5) used rabbits as experimental animals and scraped the endometrium with instruments to simulate the clinical D\&C of human endometria. It can be observed that the endometrium can be completely repaired within 3 days after simulated curettage without administration of estrogen and progesterone. This study proved that it was difficult to construct an ideal IUA animal model for non-pregnant animals with mechanical injury alone. After conducting several experiments, it was believed by investigators that it might be easier to establish an animal model of IUA by using more than 2 methods of injury. Many researchers have subsequently attempted to construct IUA animal models using a variety of methods. To form IUA, Schenker et al. (6) injected $10 \%$ formalin solution into the uterine cavity causing endometrial damage, fibrous tissue hyperplasia, and occlusion of the uterine cavity. Meanwhile, Liu et al. (7) established a rabbit IUA model after intrauterine curettage of rabbits by simulating infection with bacterial lipopolysaccharide cotton threads in the uterine cavity. Although these physical or chemical methods caused different degrees of damage to the endometrium, and even formed adhesions, they were far different from the real etiology of human IUA, and the endometrial IUA-related pathological changes were not completely faithful to the clinical reality.

In our experiments, the endometria of pregnant rats were injured by mechanical injury in a process more closely related to the etiology of human IUA. The experimental results showed that, except for the endometrial glands not 
fully recovering after the end of pregnancy on postoperative day 3 , the number of endometrial glands was reduced on the 7 th and 14th day postoperatively. After 3, 7, and 14 days, the level of endometrial fibrosis was higher, and the endometrial epithelium became thinner. Furthermore, sparse gland distribution and interstitial fibrosis were seen under the microscope, and fibrous and muscular adhesions were seen in some sections, which is consonant with the pathological and etiological manifestations of human IUA. This confirms that the pregnant rat curettage model can successfully establish a stable and accurate animal model of IUA.

\section{Mechanisms}

This study used pregnant rat curettage to establish an animal model of IUA according to the risk factors of IUA. The uterus of the pregnant rat becomes thinner and softer during pregnancy. After the embryo is cleared, the uterine cavity expands, and it is easier to imitate human's curettage procedures. Mechanical injury can fully scrape the epithelium, with part of the interstitial surface of the endometrium with the uterine wall becoming thinner during pregnancy. This causes damage to the rat endometrium and is more likely to cause IUA. If non-pregnant SD rats are selected as model animals, the diameter of uterine tube is small, the operative curette is tiny, and the uterine wall is thick, which makes it difficult to effectively scrape the endometrium and cause damage. In addition to the above, the rat endometrial recovery is fast, which contributes to the low fibrosis rate in rat IUA models. Gilman et al. studied and analysed 144 women with IUA after abortion and concluded that the mechanical suction (D\&C) and uterine enlargement, especially the uterine enlargement caused by multiple pregnancies, were risk factors for IUA. This indicates that the principle of the successful construction of the IUA model of pregnant rat curettage IUA should accord with the high-risk factors of clinical IUA (9).

Of further note, on the third day after curettage in pregnant rats, the number of glands in the study group and control group were decreased, the reason for which perhaps being that the ovarian cycle had not been fully established after the termination of pregnancy. By reviewing the literature, we realized that the complete regeneration after endometrial injury may depend on two main factors: the rapid regeneration of epithelial cells on the surface of the endometrium, covering the endometrial wound, and a lighter inflammatory response $(10,11)$. After endometrial injury, delayed epithelial regeneration on the endometrial surface may be the key factor in the formation of uterine adhesions (12). In the pregnant rat curettage model, the ovarian function has not recovered and estrogen decreases beginning from the termination of pregnancy (13). There is a lack of sufficient regenerating epithelial cells to cover the surface of the uterine cavity, resulting in stromal tissue exposure and an increase in stromal fiber activity. When combined with fibrous exudation and endometrial scar repair, this ultimately leads to IUA.

\section{Strengths and limitations}

As the cause of IUA is mainly induced by abortion, our IUA animal model is formed by scraping the uterus of pregnant rats, which is more in line with clinical practice. This is the first time that the IUA animal model has been established using a pregnant rat curettage. However, this experiment did not observe the symptoms of pregnant rats after 14 days postoperatively and did not observe their fertility outcomes, which needs to be investigated in our further studies. In addition, this experiment did not explore the relative mechanism for curettage-induced IUA. Thus, we need to conduct subsequent experiments to investigate its interrelated mechanism and generate the experimental evidence for a better understanding of IUA formation.

\section{Conclusions}

In summary, by using the pregnancy curettage-injured pregnant rat endometrium, and comparing the morphology, endometrial glandular number, the degree of fibrosis, and the expression of TGF- $\beta$, we confirmed that this method can successfully establish a stable IUA model which is more effective in mimicking the generation of human IUA.

\section{Acknowledgments}

Funding: This study was supported by the National Key Research and Development Program of China (grant no. 2018YFC1004800). The study received approval from the institutional review board of the Third Xiangya Hospital of Central South University (ID: 2019sydw0059). Bingsi Gao was supported by the China Scholarship Council (file no. 201806370178).

\section{Footnote}

Conflicts of Interest: The authors have no conflicts of interest 


\section{Page 8 of 8}

to declare.

Ethical Statement: The authors are accountable for all aspects of the work in ensuring that questions related to the accuracy or integrity of any part of the work are appropriately investigated and resolved. All experimental procedures were approved by the Animal Experimentation Ethics Committee of Third Xiangya Hospital of Central South University, Hunan, China (ID: 2019sydw0059).

\section{References}

1. Hooker A, Fraenk D, Brölmann H, et al. Prevalence of intrauterine adhesions after termination of pregnancy: a systematic review. Eur J Contracept Reprod Health Care 2016;21:329-35.

2. Mentula M, Männistö J, Gissler M, et al. Intrauterine adhesions following an induced termination of pregnancy: a nationwide cohort study. BJOG 2018;125:1424-31.

3. Azizi R, Aghebati-Maleki L, Nouri M, et al. Stem cell therapy in Asherman syndrome and thin endometrium: Stem cell- based therapy. Biomed Pharmacother 2018;102:333-43.

4. Schenker JG, Polishuk WZ. Regeneration of rabbit endometrium after cryosurgery. Obstet Gynecol 1972;40:638-45.

Cite this article as: Feng Q, Gao B, Zhao X, Huang H, Yi S, Zou L, Liu X, Xue M, Xu D. Establishment of an animal model of intrauterine adhesions after surgical abortion and curettage in pregnant rats. Ann Transl Med 2020;8(4):56. doi: 10.21037/ atm.2020.01.134

\section{Feng et al. Establishment of a D\&C IUA model in pregnant rats}

5. Polishuk WZ. Endometrial regeneration and adhesion formation. S Afr Med J 1975;49:440-2.

6. Schenker JG, Polishuk WZ. Regeneration of rabbit endometrium following intrauterine instillation of chemical agents. Gynecol Invest 1973;4:1-13.

7. Liu F, Zhu ZJ, Li P, et al. Creation of a female rabbit model for intrauterine adhesions using mechanical and infectious injury. J Surg Res 2013;183:296-303.

8. Khrouf M, Morel O, Hafiz A, et al. Evaluation of the rabbit as an experimental model for human uterine synechia. J Hum Reprod Sci 2012;5:175-80.

9. Gilman AR, Dewar KM, Rhone SA, et al. Intrauterine Adhesions Following Miscarriage: Look and Learn. J Obstet Gynaecol Can 2016;38:453-7.

10. Weiss G, Goldsmith LT, Taylor RN, et al. Inflammation in reproductive disorders. Reprod Sci 2009;16:216-29.

11. Gnainsky Y, Granot I, Aldo PB, et al. Local injury of the endometrium induces an inflammatory response that promotes successful implantation. Fertil Steril 2010;94:2030-6.

12. Bigsby RM. Control of growth and differentiation of the endometrium: the role of tissue interactions. Ann N Y Acad Sci 2002;955:110-7.

13. Yu D, Wong YM, Cheong Y, et al. Asherman syndrome-one century later. Fertil Steril 2008;89:759-79. 